\title{
Structure-aware protein solubility prediction from sequence through graph convolutional network and predicted contact map
}

Jianwen Chen ${ }^{1+}(0)$, Shuangjia Zheng ${ }^{1+}$, Huiying Zhao ${ }^{2}$ and Yuedong Yang ${ }^{1,3^{*}}$

\begin{abstract}
Protein solubility is significant in producing new soluble proteins that can reduce the cost of biocatalysts or therapeutic agents. Therefore, a computational model is highly desired to accurately predict protein solubility from the amino acid sequence. Many methods have been developed, but they are mostly based on the one-dimensional embedding of amino acids that is limited to catch spatially structural information. In this study, we have developed a new structure-aware method GraphSol to predict protein solubility by attentive graph convolutional network (GCN), where the protein topology attribute graph was constructed through predicted contact maps only from the sequence. GraphSol was shown to substantially outperform other sequence-based methods. The model was proven to be stable by consistent $R^{2}$ of 0.48 in both the cross-validation and independent test of the eSOL dataset. To our best knowledge, this is the first study to utilize the GCN for sequence-based protein solubility predictions. More importantly, this architecture could be easily extended to other protein prediction tasks requiring a raw protein sequence.
\end{abstract}

Keywords: Protein solubility prediction, Graph neural network, Predicted contact map, Deep learning

\section{Introduction}

Over the past 20 years, recombinant protein had played a vital role in biotechnology and medicine, including novel therapeutic protein drugs and antibodies [1]. Recombinant proteins are mostly produced by genetic engineering in Escherichia coli (E.coli) [2]. However, low solubility and activity of proteins expressed by E.coli limited the production efficiency even though the standard workflow and logical strategies have been widely deployed in biopharmaceutical industries [1]. According to statistics, over $30 \%$ of recombinant proteins are not soluble [3], 33-35\% of all expressed non-membrane proteins are insoluble, and $25-57 \%$ of soluble proteins are prone to aggregate at higher concentrations [4]. Moreover, the heterologous

\footnotetext{
*Correspondence: yangyd25@mail.sysu.edu.cn

${ }^{\dagger} J$ Jianwen Chen and Shuangjia Zheng contributed equal to this work

${ }^{1}$ School of Data and Computer Science, Sun Yat-Sen University, Guangzhou, China

Full list of author information is available at the end of the article
}

expression often suffers from low levels of production and insoluble recombinant proteins forming inclusion bodies. Therefore, the protein solubility plays an important role in the production of proteins for the biotechnological and pharmaceutical industries.

To enhance the performance of recombinant proteins, many experimental technologies have been developed, e.g. directed evolution, immobilization, designing better promoters, optimizing codon usage, and changing culture conditions including media and temperature [5, 6]. However, such empirical optimizations are laborintensive and time-consuming. A precise computational model is highly desired so that protein solubility can be effectively predicted. Theoretically, given an exact experimental condition (i.e. temperature, expression host, etc.), the solubility is determined mainly by its primary structure that is decided by the sequence [3]. To this end, two types of computational approaches have been proposed to predict the protein solubility: physical-based and machine/deep learning-based methods.

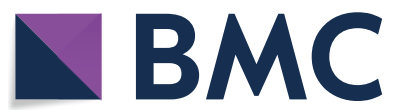

(c) The Author(s) 2021. This article is licensed under a Creative Commons Attribution 4.0 International License, which permits use, sharing, adaptation, distribution and reproduction in any medium or format, as long as you give appropriate credit to the original author(s) and the source, provide a link to the Creative Commons licence, and indicate if changes were made. The images or other third party material in this article are included in the article's Creative Commons licence, unless indicated otherwise in a credit line to the material. If material is not included in the article's Creative Commons licence and your intended use is not permitted by statutory regulation or exceeds the permitted use, you will need to obtain permission directly from the copyright holder. To view a copy of this licence, visit http://creativeco mmons.org/licenses/by/4.0/. The Creative Commons Public Domain Dedication waiver (http://creativecommons.org/publicdomain/ zero/1.0/) applies to the data made available in this article, unless otherwise stated in a credit line to the data. 
In terms of the physical-based techniques, most works $[7,8]$ focused on making use of extensive molecular dynamics simulations to evaluate the free energy difference between aggregation and solution phases. However, these methods are usually of limited accuracy due to difficulties in evaluating the conformational entropy and solvent contributions. Furthermore, these atom-level methods are sensitive to structural fluctuations and can't process protein flexibility well [9].

For the machine/deep learning techniques, several sequence-based methods have been developed for protein solubility prediction including PROSO II [10], CCSOL [5], SOLpro [11], and the scoring card method (SCM) [12]. The majority of these methods adopted the support vector machine (SVM) [13] as the core discriminative model on biologically relevant handcrafted features from protein sequences to discriminate the soluble and insoluble proteins. The newly proposed method, PaRSnIP [14] was developed by identifying correlations of protein solubilities positively with fractions of exposed residues while negatively with tri-peptide stretches containing multiple histidines. Protein-Sol [15] employed a different combination of feature weights in averaging the sequence-based local and global properties.

With the development of deep learning techniques, many end-to-end methods have been developed. DeepSol built a convolutional neural network $(C N N)$ [16] to construct non-linear high-dimensional vector spaces with essential information for predicting protein solubility [17]. ProGAN generated extra data from a Generative Adversarial Networks $(G A N)$ [18] that had been learned by the training set to improve the final performance [19]. TAPE [20] and SeqVec [21] trained a general model from a large protein database and provided a pretrained embedding for other protein downstream tasks. However, these methods are mostly based on Long Shortterm memory (LSTM) [22] or Transformer [23] and didn't utilize spatial information of protein molecules. Though our recent studies indicated that the protein structure could be well represented and the contacted structural information could be implicitly included by the residue-pairwise distance matrix through $\mathrm{CNN}[24,25]$, the information aggregated from regular Euclidean space could not fully interpret the relations between residues in contact.

In the past few years, the Graph Neural Network $(G N N)$ was raised to represent the protein structure in various deep learning-based methods and had made successes in properties prediction [26-28]. However, these methods demand experimentally obtained 3D structures that are hard to acquire for aggregation proteins and thus are not appropriated for sequence-based protein solubility prediction.
With recent developments in protein structure prediction, the prediction of protein contact map has been greatly improved according to the critical assessment of protein structure prediction (CASP) [29], which brought another way to get accurate contact structural information without using the protein 3D structures. There are quite a few predictors solved this protein by evaluating the residue-residue contact [29-31], for example, Hanson et al. [32] had developed a novel sequence-based method in predicting protein contact map and reached the stateof-the-art performance, which aimed to capture these deep, underlying relationships between residue-residue pairs in spatial dimensions for protein 'image' at each layer. Compared to other algorithms, the predicted protein contact map integrates all their advantages so that it can represent 2D structural features directly in high accuracy, enabling the construction of accurate protein graphical representations from protein sequence. And a similar constructed structure also helps us evaluate the predicted performance when compared to the possible experimental structure.

Inspired by these new development tools, we proposed a novel structure-aware method GraphSol for protein solubility prediction from the sequence by combining predicted contact maps and graph neural networks. The predicted contact maps were employed to construct protein graphs, and the attentive-based graph convolutional network made the predictions through mapping the nodes (amino acids) embedding to the graph full content embedding. We performed our model in the $e S O L$ database [33] and obtained state-of-the-art performance. To the best of our knowledge, this is the first study to make sequence-based solubility prediction for proteins through graph neural networks. Moreover, such architecture could be easily applied to extensive tasks on proteins, e.g. protein function prediction, protein-protein interaction prediction, protein folding, and drug design.

\section{Methods \\ Overview}

In this study, we convert the protein solubility prediction task as a graph-based regression problem. Given a protein sequence that consists of $L$ amino acids, the whole protein could thus be expressed as a topological attributed graph $G(F, E)$, with $F$ for the feature set of all residues (nodes) and $E$ for the residual contacts (edges) according to predicted protein contact map. Our task aims to learn a mapping function $f(\cdot)$ that inputs with predicted residual features and contact map and outputs predicted solubility with continuous scores between $[0,1] \in \mathbb{R}$ i.e. $f: G(F, E) \rightarrow[0,1]$. In this work, $f(\cdot)$ is a graph convolutional network model that aggerates nodes and edges information on the irregular graph. 


\section{Datasets}

\section{eSOL dataset}

To train our model, we employed the eSOL dataset from the previous study [34]. For completeness, we briefly describe the procedure to produce the dataset. The whole solubility database of ensemble E.coli proteins was downloaded from the $e S O L$ website [33], where the solubility was defined as the ratio of the supernatant fraction to the total fraction in the physiochemical experiments named PURE [35]. The 4132 proteins were firstly mapped to the NCBI database by gene names, and 3,144 samples were returned. We further pruned out all sequences using a strict standard that had a sequence identity $\geq 25 \%$ or $\mathrm{E}$ value $\leq 1 \mathrm{e}-6$ according to previous observation [36], and the final data included a total of 2737 protein sequences. From the dataset, $75 \%$ (2052 proteins) were randomly selected as the training set, and the remaining $25 \%$ (685 proteins) were used as the independent test.

\section{S. cerevisiae dataset}

For an external independent test, we selected another protein dataset collected by [9] from the S. cerevisiae. This dataset was derived by including 108 proteins having corresponding 3D structures. The solubilities were also measured by the cell-free expression called PURE [35] in the same external condition to reduce the influence caused by the environment.

\section{Protein representation \\ Node features}

We devised four groups of protein features that were used to train the GraphSol predictor model.

Blosum62 Instead of one-hot encoding, we have encoded residues by Blosum62 [37], which is a widely used $20 \times 20$ matrix for substitutions between 20 standard amino acid types according to alignments of homologous protein sequences. The blosum62 was shown to outperform simple one-hot encoding (results not shown), as also indicated in our previous study [38].

Physicochemical properties We utilized a set of 7 physicochemical properties for amino acid types (AAPHY7)
[39]. These features include steric parameters, hydrophobicity, volume, polarizability, isoelectric point, helix probability, and sheet probability.

Evolutionary information Evolutionarily conserved residues may contain the motifs related to protein properties (such as solubility) in biological sequences [40]. Here, we employed the position-specific scoring matrix (PSSM) and the Hidden Markov matrix (HMM). To be specific, the PSSM profile was produced by PSI-BLAST v2.7.1 [41] with the UniRef 90 sequence database after 3 iterations. The HMM profile was produced by HHblits v3.0.3 in aligning the UniClust30 profile HMM database [42] with default parameters.

Predicted structural properties Predicted structural properties are highly related to solubility in the previous study [17]. Herein, we derived the predicted structural features from SPIDER3 [43], one of the most accurate predictors. The feature group includes 14 features: (1) three probability values respectively for three secondary structure states (SS3), (2) Relative Solvent-Accessible Surface Area $(A S A)$, (3) eight values for the sine/cosine values of backbone torsion angles (phi, psi, theta, tau), and (4) Half-Sphere Exposures based on the $\mathrm{C}_{\alpha}$ atom (HSE-up and HSE-down).

Finally, these feature groups constructed the node feature matrix $\mathrm{X} \in \mathbb{R}^{L \times 94}$ with $L$ representing the length of a protein sequence. Table 1 listed all node feature groups with their dimensions. All data were standardized to zero mean and unit variance before input into the neural network.

\section{Edge features}

In order to construct the edges for the protein attribute graph representation, we make predictions of the protein contact map from a sequence by SPOT-Contact [32], which outputs the possibilities to form contacts between all residue pairs in one protein. In default, the graph is a fully connected graph constructed with each edge valued as the predicted contact probability of the corresponding residue pair. As the actual number of contacts in a protein is

Table 1 Node features and dimensions

\begin{tabular}{llc}
\hline Group & Node features & Names \\
\hline 1 & Blocks substitution matrix & BLOSUM62 \\
2 & Physicochemical properties & AAPHY7 \\
3 & Position-specific scoring matrix & PSSM \\
& Hidden markov matrix & HMM \\
4 & Structural properties predicted by SPIDER3 & SPIDER3 \\
\hline
\end{tabular}


approximately proportional to the protein sequence length, we also test constructing the protein attribute graph by setting up edges for $\alpha \times \mathrm{L}$ residual pairs with the highest predicted contact probability, as also used in the CASP [29]. The $\alpha=1 \sim 7$ was utilized as suggested by [44]. Herein, we tested two schemes to construct each selected edge by setting the value as " 1 " (discrete) and the predicted contact probability (continuous), respectively. The 2-hop neighbored residues in a sequence are always connected with the value " 1 ". Notably, though the fully connected graph (default mode) was shown to perform the best according to our results, the partial edges decrease the computational and memory complexity from $\mathrm{O}\left(\mathrm{L}^{2}\right)$ to $\mathrm{O}(\mathrm{L})$.

\section{Deep learning framework}

Our graph-based model consists of three parts. As shown in Fig. 1, the first part is a graph convolution network $(\mathrm{GCN})$, which aggregates protein structural information from its nodes and edges during iterations. The second part is a self-attention pooling layer, which transforms the node hidden state with varied sizes to the graph representation vector with a fixed size. Finally, this fix-sized vector goes through some full connection layers to predict the protein solubility.

\section{Graph convolution network}

Given a protein sequence with $L$ amino acids, the protein is represented by the feature matrix $X \in \mathbb{R}^{L \times f}$ for nodes and contact matrix $A \in \mathbb{R}^{L \times L}$ for edges with $f$ as the dimension of features for nodes. Our graph convolution network takes the following formula [45]:

$$
G^{(l+1)}=\sigma\left(\tilde{\sim}^{-1} \tilde{A} G^{(l)} W^{(l)}\right),
$$

where $\widetilde{A}=A+I_{L}$ is the adjacency matrix by adding the edge matrix $A$ determined by the predicted contact map and the identity matrix $I_{L}$ for self-loops. $\widetilde{D} \in \mathbb{R}^{L \times L}$ is a diagonal degree matrix with $\widetilde{D}_{i i}=\sum_{k} A_{i k}$ that is used to normalize $\tilde{A}$ to sum up to 1.0 in each row. $G^{(l)} \in \mathbb{R}^{L \times f}$ is the activation hidden matrix in the $l^{\text {th }}$ layers with the initial state $G^{(0)}=X$ here. $W^{(l)} \in \mathbb{R}^{f \times f^{\prime}}$ is a weight matrix of layer-specific trainable parameters to map the iteration to a lower dimension rich-information space with a size of $f^{\prime} . \sigma$ denotes a nonlinear activation function and we use the $\operatorname{ReLU}(\cdot)$ function here. After each GCN layer, a normalization layer is added to accelerate the convergence of the GCN layers as well as reduce the overfitting problem. The final output of these GCN layers are integrated as

$$
M=\left(v_{1}, v_{2}, \ldots, v_{L}\right),
$$

where $v_{i}$ is a $p$ dimensional vector token embedding for the $i t h$ node. As a result, $M$ is a $2 \mathrm{D}$ matrix to integrate all token embeddings with $\mathbb{R}^{L \times p}$.

\section{Self-attention pooling}

Note that the output matrix $\mathrm{M}$ is dependent on the protein length, which is a variable scale. To obtain a fixed size of protein representation, a readout transformation is essential to eliminate the size variance and sequence permutation variance [46]. Herein, we employ the self-attention mechanism [47], which computes the weight coefficients $T \in \mathbb{R}^{r \times L}$ with $r$ for the number of attention groups by:

$$
T=\operatorname{SoftMax}\left(\boldsymbol{W}_{2} \tanh \left(W_{1} M^{T}\right)\right),
$$

where $M^{T}$ is the transposition of $M \in \mathbb{R}^{L \times p}$. $W_{1} \in \mathbb{R}^{q \times p}$ and $\boldsymbol{W}_{2} \in \mathbb{R}^{r \times q}$ are two learned attention matrices with the hyper-parameters $q$ and $r$. The SoftMax function standardizes each row of the computed weights, to sum up to 1 . Intuitively, the $r$ groups of attention coefficients assess the associations of each residue with the solubility from different views. Thus, we extract the overall features by multiplying $\mathrm{T}$ and $\mathrm{M}$, and average all $r$ groups of attention coefficients for the final graph representation $H \in \mathbb{R}^{1 \times p}$ by

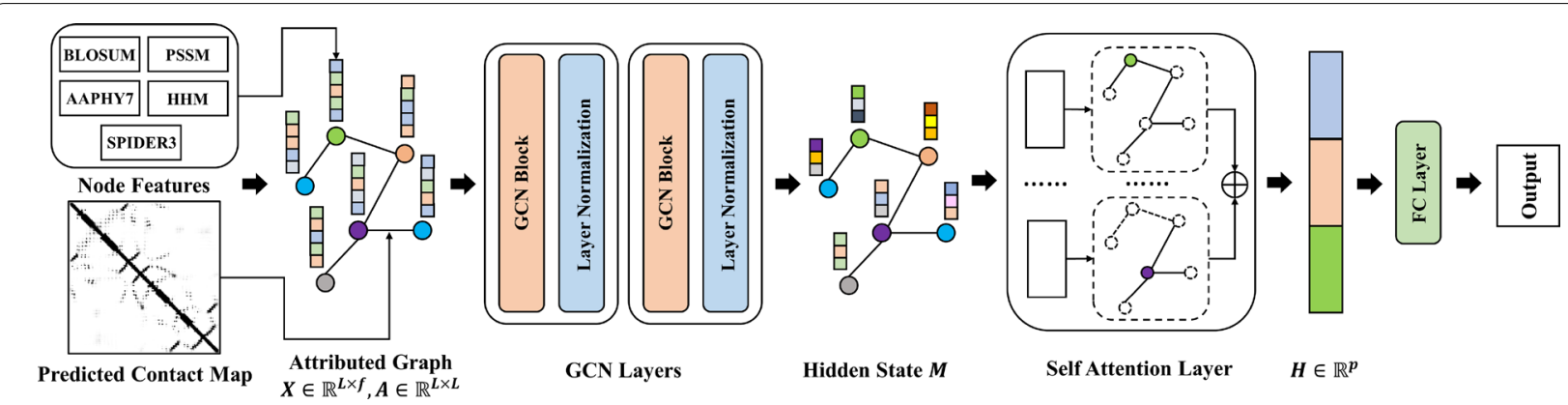

Fig. 1 The overall framework of the GraphSol model. The compiled node features $(X)$ and contact maps (A) were utilized to construct the GCN networks, which were convoluted by two GCN blocks and layer normalizations for hidden states (M). The hidden states were converted by the self-attention layer as a fixed length of the vector $(H)$, which were input to the MLP to make the final prediction 


$$
H=\frac{1}{r} \sum_{k=1}^{r}(T M)_{k}
$$

\section{Multilayer perceptron}

The output of self-attention pooling was input to the multilayer perceptron (MLP) to predict the solubility $S$ by

$$
S=\operatorname{Sigmoid}\left(\mathbf{W}_{3} H^{\mathrm{T}}+\mathbf{b}\right),
$$

where $\mathbf{W}_{3} \in \mathbb{R}^{1 \times \mathrm{p}}$ is the weight matrix and $\mathrm{b} \in \mathbb{R}$ is the bias item. The sigmoid function maps the value to $(0,1)$ for solubility prediction.

\section{Training and evaluation}

\section{Hyper-parameters tuning}

Our model for solubility prediction includes multiple hyperparameters. We tested crucial hyperparameters and the range of values as follows:

GCN layers A higher number of GCN layers means the wider and deeper information aggregated from the edge and node features. However, excessive layers would cause a decrease in final predicted accuracy due to vanishing gradients. Therefore, it is crucial to keep a balance between the layers and the algorithm complexity. We tested the following settings $(1,2,3,4)$ and found $2 \mathrm{GCN}$ layers to be the optimal value after tuning on the validation sets.

GCN middle dimensions These hyper-parameters control the channel dimensions in all stacking GCN layers including the final GCN layer. They influence the matrices that are transferred into self-attention pooling to identify the key soluble fragment of the protein. Therefore, we should construct a suitable size for matrices in liberating the rich-information regions as well as the distinguishability of different proteins. As a result, the optimal parameters are 64 dimensions for the last layer, and 256 for others.

Attention heads The attention heads provide weight coefficients to focus on key residues for the solubility prediction, and different heads enable the attention of multiple regions from different views. We tested the number of attention heads from 1 to 10 and found 4 attention heads provided the best performance and the least calculation in the validations.

Besides, the models were trained for different epochs using Adam optimizer [48]. Additional file 1: Table S2 showed the optimal hyper-parameters by the grid search.

\section{Cross-validation and independent test}

We performed the fivefold cross-validation on the training dataset. That is, proteins in the training dataset were separated into five parts (folds). In each round four folds were employed to train a model that was evaluated on the left one-fold. This process was repeated five times, and the performances of five predictions were averaged as the validation performance. To reduce fluctuations by the random splitting of fivefold, we have split the training set with five random seeds and took an average of final performances. The validations were used to optimize all hyper-parameters. After fine-tuning the optimal hyperparameters, a model was trained by all training dataset and independently tested on the two independent test datasets.

\section{Evaluation indicators}

The neural network was trained to minimize the root of mean squared error (RMSE), and the coefficient of determination $\left(R^{2}\right)$ was used to evaluate our models and optimize the hyper-parameters. Since many compared methods [5, 14, 15, 17] have been developed to classify whether a protein is soluble or not, we also separate all proteins into two classes by a threshold of 0.5 for the predicted and actual solubility. Statistically speaking, the definition of solubility mentioned before supports us regarded the soluble fraction of proteins as the soluble probabilities from other perspectives. Based on this setting, the models were evaluated by the area under the Receiver Operating Characteristic (ROC) curve (AUC), accuracy, precision, recall, and F1 defined as:

$$
\begin{aligned}
& \text { Precision }=T P /(T P+F P) \\
& \text { Recall }=T p /(T P+F N) \\
& F_{1}=2 \times(\text { Precision } \times \text { Recall }) /(\text { Precision }+ \text { Recall })
\end{aligned}
$$

where TP, FP, TN, and FN denote the numbers of true positives (soluble proteins), false positives (non-soluble protein predicted as soluble), true negatives, and false negatives, respectively.

\section{Results \\ Performances on the fivefold cross-validation and independent test}

We investigated the performance of the GraphSol model on the $e S O L$ dataset. As shown in Table 2, We obtained $\mathrm{R}^{2}$ values of $0.476 \pm 0.014$ and 0.483 for the fivefold cross-validation (CV) and independent test, respectively. When separating the dataset into two 
Table 2 The $\mathrm{R}^{2}$ between the actual solubility scores and those predicted by GraphSol based on individual feature groups, removing each feature group from the final GraphSol model, and recursively adding feature groups according to their importance, respectively

\begin{tabular}{lllllllll}
\hline Feature groups $^{\mathbf{a}}$ & $\mathbf{C V}^{\mathbf{d}}$ & Ind. test & Feature groups & $\mathbf{C V}^{\mathbf{d}}$ & Ind. test & Features groups & $\mathbf{C V}^{\mathbf{d}}$ & Ind. test $^{\mathbf{c}}$ \\
\hline- & - & - & GraphSol & $0.476 \pm 0.014$ & 0.483 & - & - & - \\
BLOSUM & $0.329 \pm 0.016$ & 0.317 & - BLOSUM & $0.460 \pm 0.011$ & 0.465 & BLOSUM & $0.329 \pm 0.016$ & 0.317 \\
AAPHY7 & $0.293 \pm 0.014$ & 0.289 & - AAPHY7 & $0.465 \pm 0.012$ & 0.479 & + SPIDER3 & $0.413 \pm 0.012$ & 0.409 \\
PSSM & $0.333 \pm 0.012$ & 0.332 & - PSSM & $0.457 \pm 0.017$ & 0.467 & + PSSM & $0.456 \pm 0.011$ & 0.453 \\
HMM & $0.337 \pm 0.015$ & 0.341 & - HMM & $0.455 \pm 0.016$ & 0.458 & + HMM & $0.465 \pm 0.012$ & 0.479 \\
SPIDER3 & $0.231 \pm 0.019$ & 0.227 & - SPIDER3 & $0.428 \pm 0.018$ & 0.449 & + AAPHY7 & $0.476 \pm 0.014$ & 0.483 \\
\hline
\end{tabular}

Italic values indicate the performance of using all feature groups in our model

a Performances based on individual feature groups

b by removing each feature group from all feature

c by adding feature groups recursively

d Performances by the fivefold cross-validation

discrete states (soluble or not soluble) by a cutoff of 0.5 , the Area Under Curve (AUC) values are 0.855 and 0.866 for the fivefold CV and independent test, respectively (Additional file 1: Figure S1). The similar results by the $\mathrm{CV}$ and independent test indicated the robustness of the GraphSol model.

In order to indicate the importance of feature groups, we assessed the performances by 3 ways in the ablation study. As shown in Table 2, when the individual feature group was used as the node features, the HMM feature yielded the highest $R^{2}$ with a value of 0.341 in the independent test. The other evolution-based feature (PSSM) performed similarly but slightly worse than HMM. Not surprisingly, the BLOSUM feature didn't perform well with $\mathrm{R}^{2}$ of 0.317 , but better than the AAPHY7. The predicted structural feature group (SPIDER3) made the worst performance with $R^{2}$ of 0.243 . When removing an individual group, on the contrary, the removal of SPIDER3 led to the greatest drop from 0.483 to 0.449 . This is likely because SPIDER3 uniquely provided structural information, while other features have supplementary alternatives. Though PSSM and HMM similarly represent evolution information, their removals still caused decreases in performances and generally, HMM is shown more important. The removal of AAPHY7 caused the smallest drop, which is understandable because this feature is a seven-dimensional matrix that is smaller than other feature groups. When we evaluated the model by adding the feature groups recursively, the model showed incremental performances with the addition of each feature group. An interesting fact was that the performance sharply increased from 0.317 to 0.409 after adding SPIDER3 features, which agreed with the relationship between solubility and structural features such as the secondary structure and solvent accessible area.

\section{Evaluating the impact of predicted protein contact map}

The previous results were based on a fully connected graph with edges weighted according to predicted contact probabilities. As there are a limited number of actual contacts between residue pairs, we tested assigning edges between top $\alpha \times \mathrm{L}$ residue pairs with the highest predicted contacting probabilities. As shown in Fig. 2, when not using the predicted contact map $(\alpha=0)$, i.e. no edges were assigned except between 2-hops neighbored residues, the model achieved $R^{2}$ of 0.462 . With the increase of $\alpha$, the $R^{2}$ has a steady increase followed by a sharp increase from $2 \times \mathrm{L}$ to $3 \times \mathrm{L}$ with the $\mathrm{R}^{2}$ of 0.474 . Then a slight but continuous growth was observed with an increase of $\alpha$. The highest $R^{2}$ of 0.483 was obtained when all residues were connected with the respective predicted probability.

By comparison, we tested the connectivity by discretely assigning all connected edges as 1 with other pairs not connected and labeled to 0 . As expected, the $\mathrm{R}^{2}$ increased with $\alpha$ from 0 to 3 , indicating that the pairs are helpful for the prediction. Afterward, the $\mathrm{R}^{2}$ started to decrease

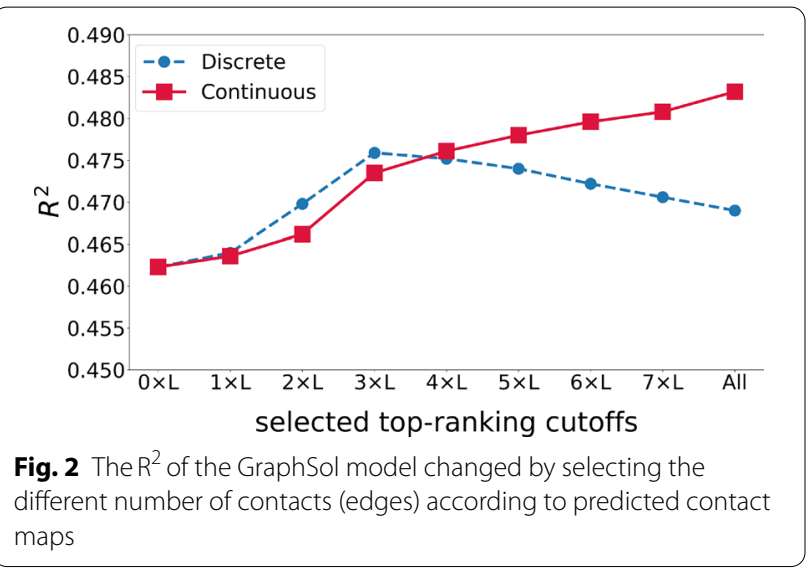


likely due to the increase of inaccurately predicted contact pairs information. Interestingly, we found there are close $\mathrm{R}^{2}$ for the discrete group and continuous group at $\alpha$ nearly to 4 , which indicates that they may promote equivalent information for the final prediction.

\section{Comparisons with other methods}

Our GraphSol model was compared with state-of-theart methods. To avoid the impacts caused by data, all machine learning or deep learning-based models were retrained and tested on the same train and test dataset, respectively. As shown in Table 2, GraphSol consistently obtained the best results by all measurements as a single method with $R^{2}=0.483$. Even if we didn't use predicted contact maps i.e. no edges were assigned except between 2-hops neighbored residues, the GraphSol (no-contact) ranked the 2nd that yielded slightly higher $\mathrm{R}^{2}$ than our self-implemented LSTM model (0.462 vs 0.458$)$. This similar result was expected since GraphSol (no-contact) utilized the neighbor residue's information explicitly and the LSTM model obtained the same information implicitly. And SPIDER3 played a key role in providing accurate structural information (0.458 vs 0.449$)$, we inferred this improvement may come from the message in the atomlevel rather than the contact in the edge-level. For other methods, TAPE [20] and SeqVec [21], two transfer-learning methods, achieved the highest $\mathrm{R}^{2}=0.461$ and the second-highest $\mathrm{R}^{2}=0.458$, which lower than GraphSol $\left(R^{2}=0.483\right)$ but higher than other non-transfer-learning methods. ProGAN [19], a GAN network-based method, achieved $\mathrm{R}^{2}=0.442$, which is $4 \%$ lower than TAPE and GraphSol(no-contact). DeepSol, a CNN-based network, achieved $R^{2}$ of 0.434 , which is close to the ProGAN. Other Machine learning techniques didn't perform well with $\mathrm{R}^{2}$ ranging from 0.214 to 0.411 . Figure 3 shows the actual solubility as a function of predicted values by four methods. We found that the deep learning-based methods fitted more accuracy in the region [0.2,0.4], especially the ProGAN and GraphSol model, and our model performed better in the region of 0.2 .

As most of the other methods were designed for predicting discrete states, we also turn the problem into the 2-state classification task. When using a threshold of 0.5 to define soluble proteins or not, the GraphSol model achieved the best performances with an AUC of 0.866, F-measure of 0.732 , and accuracy of 0.779 , which are at least $2 \%$ better than the best of other methods. Figure 4 compared the ROC curves for six methods, and we can find that the curve of GraphSol mostly locates on the top. We also tested how accuracy varies as a function of the threshold which defined a soluble class and the trends were similar (Additional file 1: Figures S2, S3).

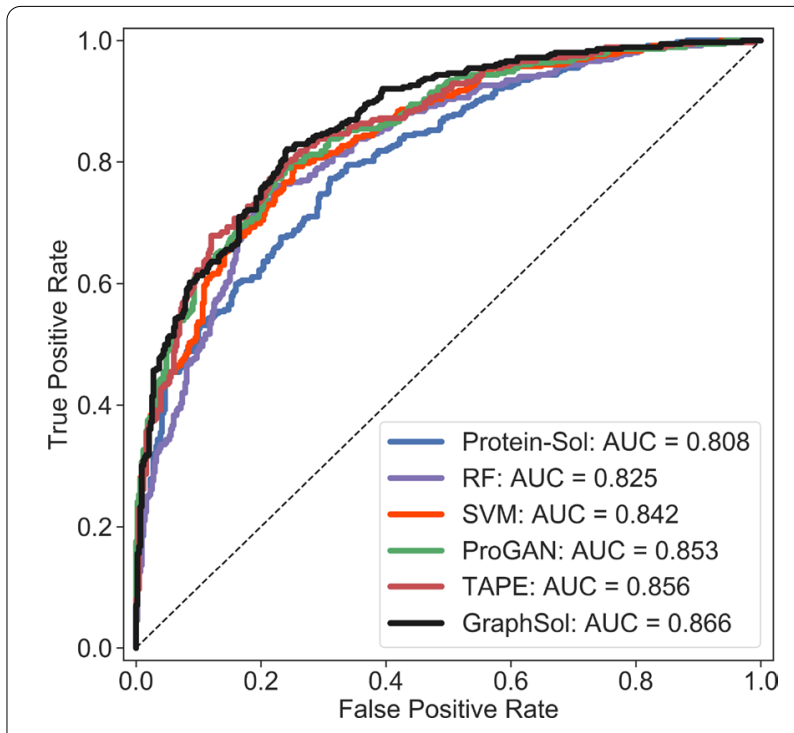

Fig. 4 Comparisons of the areas under the receiver operating curve (AUC) on the eSOL test set

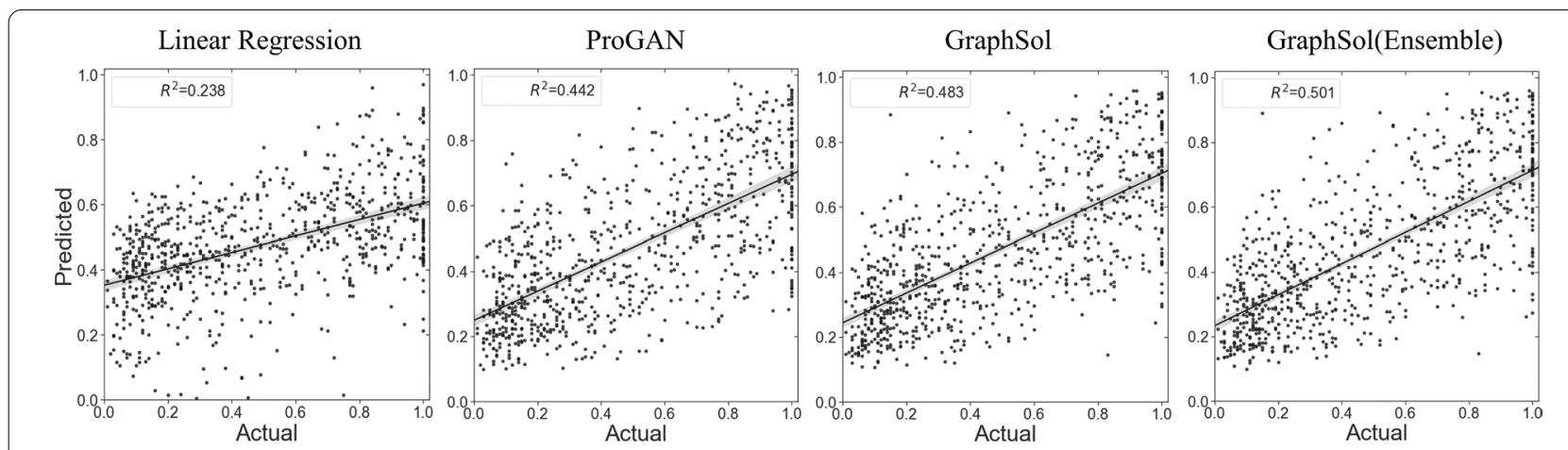

Fig. 3 Comparison between actual solubility scores and those predicted by four sequence-based methods on the independent test. The line is the linear least-square fitting to the actual values with the shadow as the $95 \%$ confidence intervals for the regressions 
We noticed that GraphSol showed fluctuations in the fivefold cross-validation, and thus we built an ensemble model by averaging the outputs of 5 trained models during CV on the test set. The GraphSol (Ensemble) model was found to further improve the performance by a margin of $3 \%$ to 0.501 in $\mathrm{R}^{2}$. Other indicators also got varied increases (Table 3).

Moreover, we made comparisons of all methods on the other external S. cerevisiae test set. Here, we employed the $e S O L$ training dataset to train the model, and have excluded sequences with identity $>25 \%$ to the $S$. cerevisiae test dataset. As shown in Table 4, GraphSol model yielded $R^{2}$ of 0.358 which is much higher than other sequence-based methods. In comparison, the DeepSol and ProGAN achieved an $\mathrm{R}^{2}$ below 0.1. And our ensemble method could further improve GraphSol by $3.9 \%$, consistent with the previous results on the $e S O L$ dataset. It is noted that all methods achieved lower $\mathrm{R}^{2}$ on this dataset. This is likely because this dataset is more challenging with low homology, as the Solart method was reported to yield $R^{2}$ of 0.422 even with the use of experimental structures. This method wasn't directly compared because most proteins didn't contain experimental structures as also indicated in the $e S O L$ dataset. We also consider the quality of the predicted contact map with the best discrete state $3 \times \mathrm{L}$ and $\mathrm{C}_{\alpha}$ distance less than $7.5 \AA$. As a result, the average values of recall and precision are 0.699 and 0.439 , respectively. The poor quality of the predicted contact map may lead to the lower $\mathrm{R}^{2}$.

\section{Case study}

To further illustrate our method, we took the Peptidyllysine $\mathrm{N}$-acetyltransferase $y j a B$ gene (PDB id: $2 \mathrm{kcw}$ )
Table 4 Comparisons of different methods on the S. cerevisiae test set

\begin{tabular}{ll}
\hline Solubility predictors & $\mathbf{R}^{\mathbf{2}}$ \\
\hline GraphSol (ensemble) & 0.372 \\
GraphSol & 0.358 \\
ccSol $^{a}$ & 0.302 \\
Protein-Sol $^{\mathrm{a}}$ & 0.281 \\
$\mathrm{CamSol}^{\mathrm{a}}$ & 0.160 \\
DeepSol $^{\mathrm{a}}$ & 0.090 \\
ProGAN $^{\mathrm{b}}$ & 0.084 \\
\hline Italic values indicate the best performance of our single model and ensemble $^{\text {model, respectively }}$ & \\
${ }^{\text {a }}$ Results produced by [7] & \\
${ }^{\mathrm{b}}$ Results produced by us &
\end{tabular}

that consisted of 147 residues as an example, which showed the lowest RMSE during the independent test. We calculated the $C_{\alpha}$ distance between all residue pairs as the actual contact map. As shown in Fig. 5, there are 360 residue pairs with $C_{\alpha}$ distance less than $7.5 \AA$ on the actual contact map of the protein. The prediction corresponded to a precision of 0.745 to cover $75.3 \%$ of actual contacts (Additional file 1: Table S4). This highquality prediction of the residue pairs enabled the accurate construction of the protein attitude graph and the solubility prediction. Besides, when compared to the actual solubility of 0.87 , the GraphSol model made an accurate prediction of 0.864 and 0.857 by using the continuous and $3 \times \mathrm{L}$ discrete predicted contact map, respectively. This similar tendency between Fig. 2 and Additional file 1: Figure S4 also indicated the effectiveness of our method.

Table 3 Comparisons of different methods on the eSOL test dataset

\begin{tabular}{|c|c|c|c|c|c|c|c|}
\hline Models & RMSE & $R^{2}$ & Accuracy & Precision & Recall & F1 & AUC \\
\hline K-nearest neighbor & 0.284 & 0.214 & 0.691 & 0.737 & 0.486 & 0.586 & 0.776 \\
\hline Linear regression & 0.280 & 0.240 & 0.707 & 0.685 & 0.642 & 0.663 & 0.777 \\
\hline Random forest & 0.255 & 0.370 & 0.760 & 0.750 & 0.690 & 0.729 & 0.825 \\
\hline Protein-Sol & 0.253 & 0.376 & 0.714 & 0.689 & 0.688 & 0.693 & 0.808 \\
\hline XGboost & 0.252 & 0.385 & 0.756 & 0.748 & 0.690 & 0.718 & 0.829 \\
\hline Support vector machine & 0.246 & 0.411 & 0.761 & 0.763 & 0.684 & 0.721 & 0.842 \\
\hline DeepSol & 0.241 & 0.434 & 0.763 & 0.771 & 0.738 & 0.695 & 0.845 \\
\hline ProGAN & 0.237 & 0.442 & 0.763 & 0.770 & 0.676 & 0.720 & 0.853 \\
\hline SeqVec & 0.236 & 0.458 & 0.767 & 0.754 & 0.715 & 0.734 & 0.858 \\
\hline TAPE & 0.235 & 0.461 & 0.764 & 0.774 & 0.710 & 0.730 & 0.856 \\
\hline LSTM (All node features) & 0.236 & 0.458 & 0.765 & 0.748 & 0.677 & 0.730 & 0.855 \\
\hline GraphSol (No contact) & 0.235 & 0.462 & 0.763 & 0.710 & 0.676 & 0.729 & 0.853 \\
\hline GraphSol & 0.231 & 0.483 & 0.779 & 0.775 & 0.693 & 0.732 & 0.866 \\
\hline GraphSol (Ensemble) & 0.227 & 0.501 & 0.782 & 0.790 & 0.702 & 0.743 & 0.873 \\
\hline
\end{tabular}

Italic values indicate the performance of our purposed model

Bold italic values indicate the performance of our ensemble model by using all folds of models to make a final prediction 

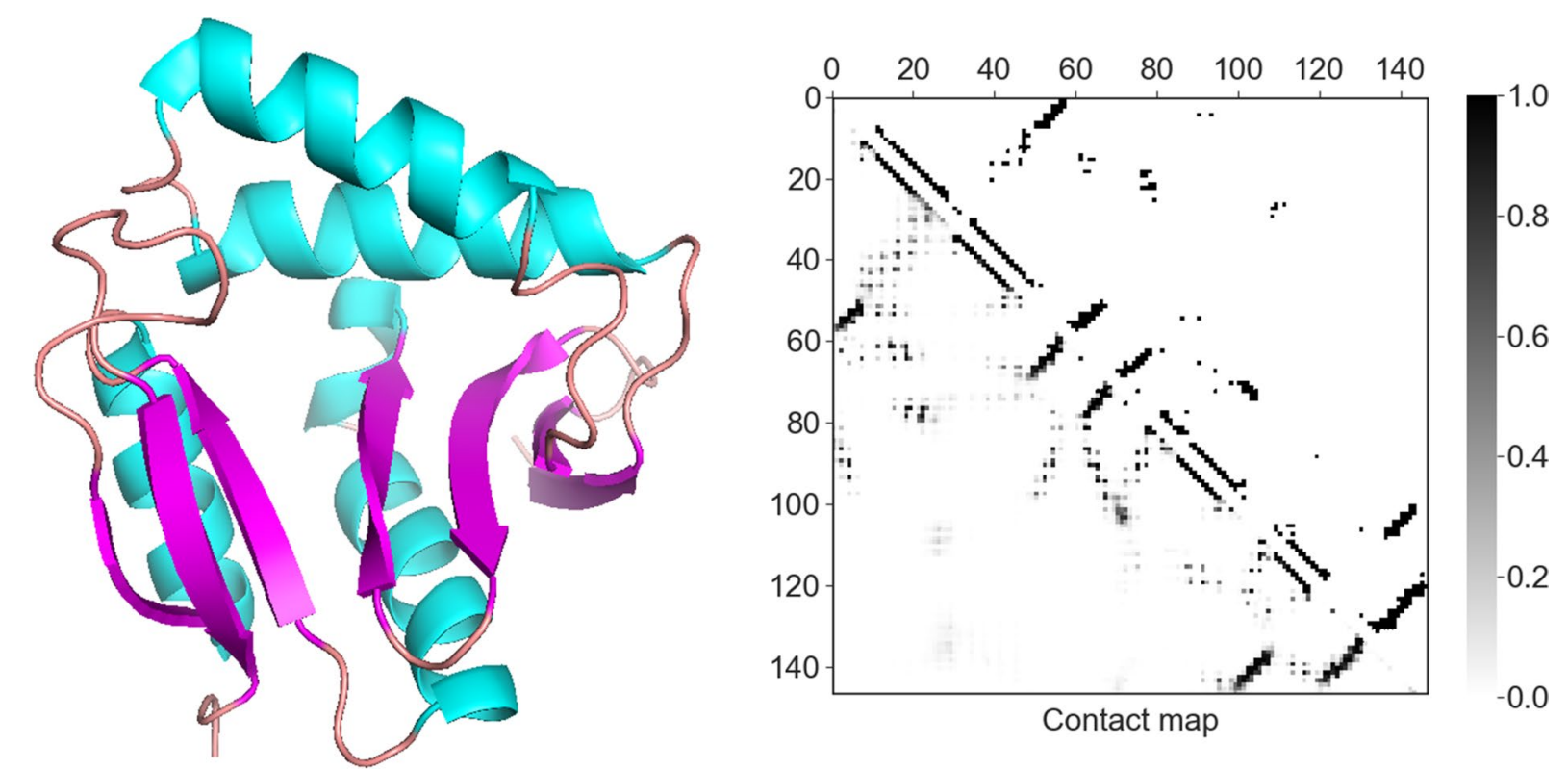

Fig. 5 the 3D structure and the predicted (the left down triangular matrix) and actual (the right top one) contact map of the selected case (the Peptidyl-lysine N-acetyltransferase yjaB gene, PDB ID: 2kcw)

\section{Conclusions}

In this study, we introduce the GraphSol model, a novel sequence-based solubility predictor. Compared to other methods, we utilized predicted protein contact maps that played a key role in bridging protein topology attribute and attentive graph neural network. We found that the predicted contact probabilities between residues are better to represent the pairwise relations than discrete states. In the future, such a method is potentially useful to protein attribute predictions including protein function, protein-protein interaction, protein folding, and drug design.

\section{Supplementary Information}

The online version contains supplementary material available at https://doi. org/10.1186/s13321-021-00488-1.

Additional file 1: Table S1. The abbreviations list in the paper. Table S2. Important hyperparameters were used in the GraphSol model. Table S3. The performance of fivefold CV and independent test in the Grid Search of each parameter. Table S4. The confused matrix between the predicted contact map and actual contact map of the protein sequence in the case study. Figure S1. Comparison of the area under the receiver operating curve (AUC) of GraphSol models on the fivefold CV and independent test. Figure S2. Comparison of the precision-recall curve of GraphSol models with the other 5 methods on the independent test. Figure S3. Comparison of the accuracy by setting different cutoff for the soluble class with the other 6 methods on the independent test. Figure S4. The solubility prediction of the protein sequence that was produced by gene yjaB, with the GraphSol model changed by selecting different thresholds according to predicted protein contact maps.

\section{Acknowledgments}

This study has been supported by the National Key R\&D Program of China (2020YFB020003), National Natural Science Foundation of China (61772566), Guangdong Key Field R\&D Plan (2019B020228001 and 2018B010109006), Introducing Innovative and Entrepreneurial Teams (2016ZT06D211), Guangzhou S\&T Research Plan (202007030010).

\section{Authors' contributions}

JC, SZ, and YY contributed to the concept and implementation. JC and SZ COdesigned experiments. JC was responsible for programming. All authors contributed to the interpretation of results. JC, SZ, and YY wrote the manuscript. All authors reviewed the final manuscript. All authors read and approved the final manuscript.

\section{Available of data and materials}

The source code of GraphSo/ is available at https://github.com/jcchan23/ GraphSol.

\section{Competing interests}

The authors declare that they have no competing interests.

\section{Author details}

${ }^{1}$ School of Data and Computer Science, Sun Yat-Sen University, Guangzhou, China. ${ }^{2}$ Sun Yat-Sen Memorial Hospital, Sun Yat-Sen University, Guangzhou, China. ${ }^{3}$ Key Laboratory of Machine Intelligence and Advanced Computing (Sun Yat-Sen University), Guangzhou 510000, China.

Received: 21 October 2020 Accepted: 20 January 2021

Published online: 08 February 2021

\section{References}

1. Habibi N, Hashim SZM, Norouzi A, Samian MR (2014) A review of machine learning methods to predict the solubility of overexpressed recombinant proteins in Escherichia coli. BMC Bioinform 15(1):134

2. Chan W-C, Liang P-H, Shih Y-P, Yang U-C, Lin W-C, Hsu C-N (2010) Learning to predict expression efficacy of vectors in recombinant protein production BMC bioinform 11(S1):S21 
3. SamakT, Gunter D, Wang Z: Prediction of protein solubility in E. coli. In: 2012 IEEE 8th international conference on E-science. New York: IEEE; 2012. p. 1-8.

4. Fang Y, Fang J (2013) Discrimination of soluble and aggregation-prone proteins based on sequence information. Mol BioSyst 9(4):806-811

5. Agostini F, Vendruscolo M, Tartaglia GG (2012) Sequence-based prediction of protein solubility. J Mol Biol 421(2-3):237-241

6. Madhavan A, Sindhu R, Binod P, Sukumaran RK, Pandey A (2017) Strategies for design of improved biocatalysts for industrial applications. Biores Technol 245:1304-1313

7. Tjong H, Zhou H-X (2008) Prediction of protein solubility from calculation of transfer free energy. Biophys J 95(6):2601-2609

8. De Simone A, Dhulesia A, Soldi G, Vendruscolo M, Hsu STD, Chiti F, Dobson CM (2011) Experimental free energy surfaces reveal the mechanisms of maintenance of protein solubility. Proc Natl Acad Sci 108(52):21057-21062

9. Hou Q, Kwasigroch JM, Rooman M, Pucci F (2020) SOLart: a structure-based method to predict protein solubility and aggregation. Bioinformatics 36(5):1445-1452

10. Smialowski P, Doose G, Torkler P, Kaufmann S, Frishman D (2012) PROSO I-a new method for protein solubility prediction. FEBS J 279(12):2192-2200

11. Magnan CN, Randall A, Baldi P (2009) SOLpro: accurate sequence-based prediction of protein solubility. Bioinformatics 25(17):2200-2207

12. Huang H-L, Charoenkwan P, Kao T-F, Lee H-C, Chang F-L, Huang W-L, Ho S-J, Shu L-S, Chen W-L, Ho S-Y (2012) Prediction and analysis of protein solubility using a novel scoring card method with dipeptide composition. BMC bioinform 13:S3

13. Suykens JAK (2002) Least squares support vector machines. World Scientific, Singapore

14. Rawi R, Mall R, Kunji K, Shen C-H, Kwong PD, Chuang G-Y (2018) PaRSnIP: sequence-based protein solubility prediction using gradient boosting machine. Bioinformatics 34(7):1092-1098

15. Hebditch M, Carballo-Amador MA, Charonis S, Curtis R, Warwicker J (2017) Protein-Sol: a web tool for predicting protein solubility from sequence. Bioinformatics 33(19):3098-3100

16. LeCun Y. LeNet-5, convolutional neural networks. 2015; 20(5):14. http://yann. lecun.com/exdb/lenet

17. Khurana S, Rawi R, Kunji K, Chuang G-Y, Bensmail H, Mall R (2018) DeepSol: a deep learning framework for sequence-based protein solubility prediction. Bioinformatics 34(15):2605-2613

18. Goodfellow IJ, Pouget-Abadie J, Mirza M, Xu B, Warde-Farley D, Ozair S, Courville A, Bengio Y. Generative adversarial networks. https://arxiv.org/ $\mathrm{abs} / 1406.2661$

19. Han X, Zhang L, Zhou K, Wang X (2019) ProGAN: Protein solubility generative adversarial nets for data augmentation in DNN framework. Comput Chem Eng 131:106533

20. Rao R, Bhattacharya N, Thomas N, Duan Y, Chen P, Canny J, Abbeel P, Song YS (2019) Evaluating protein transfer learning with TAPE. Adv Neural Inf Process Syst 32:9689-9701

21. Heinzinger M, Elnaggar A, Wang Y, Dallago C, Nechaev D, Matthes F, Rost B (2019) Modeling aspects of the language of life through transfer-learning protein sequences. BMC Bioinform 20(1):723

22. Hochreiter S, Schmidhuber J (1997) Long short-term memory. Neural Comput 9(8):1735-1780

23. Vaswani A, Shazeer N, Parmar N, Uszkoreit J, Jones L, Gomez AN, Kaiser Ł, Polosukhin I. Attention is all you need. In: Advances in neural information processing systems; 2017. p. 5998-6008. https://arxiv.org/abs/1706.03762

24. Chen S, Sun Z, Lin L, Liu Z, Liu X, Chong Y, Lu Y, Zhao H, Yang Y (2019) To improve protein sequence profile prediction through image captioning on pairwise residue distance map. J Chem Inf Model 60(1):391-399

25. Zheng S, Li Y, Chen S, Xu J, Yang Y (2020) Predicting drug-protein interaction using quasi-visual question answering system. Nat Mach Intell 2(2):134-140

26. Gligorijević V, Barot M, Bonneau R (2018) deepNF: deep network fusion for protein function prediction. Bioinformatics 34(22):3873-3881

27. Zamora-Resendiz R, Crivelli S. Structural learning of proteins using graph convolutional neural networks. bioRxiv; 2019. p. 610444.

28. Gligorijevic V, Renfrew PD, KosciolekT, Leman JK, Berenberg D, Vatanen T, Chandler C, Taylor15 BC, Fisk10 IM, Vlamakis H. Structure-based protein function prediction using graph convolutional networks. https://www.biorx iv.org/content/10.1101/786236v2.abstract

29. Schaarschmidt J, Monastyrskyy B, Kryshtafovych A, Bonvin AM (2018) Assessment of contact predictions in CASP12: co-evolution and deep learning coming of age. Proteins Struct Funct Bioinform 86:51-66

30. Wang S, Sun S, Xu J (2018) Analysis of deep learning methods for blind protein contact prediction in CASP12. Proteins Struct Funct Bioinform 86:67-77

31. Adhikari B, Hou J, Cheng J (2018) DNCON2: improved protein contact prediction using two-level deep convolutional neural networks. Bioinformatics 34(9):1466-1472

32. Hanson J, Paliwal K, Litfin T, Yang Y, Zhou Y (2018) Accurate prediction of protein contact maps by coupling residual two-dimensional bidirectional long short-term memory with convolutional neural networks. Bioinformatics 34(23):4039-4045

33. Niwa T, Ying B-W, Saito K, Jin W, Takada S, Ueda T, Taguchi H (2009) Bimodal protein solubility distribution revealed by an aggregation analysis of the entire ensemble of Escherichia coli proteins. Proc Natl Acad Sci 106(11):4201-4206

34. Han X, Wang X, Zhou K (2019) Develop machine learning-based regression predictive models for engineering protein solubility. Bioinformatics 35(22):4640-4646

35. Shimizu Y, Kanamori T, Ueda T (2005) Protein synthesis by pure translation systems. Methods 36(3):299-304

36. Li Z, Yang Y, Zhan J, Dai L, Zhou Y. Energy functions in de novo protein design: current challenges and future prospects. 2013. https://www.annua Ireviews.org/doi/full/10.1146/annurev-biophys-083012-130315

37. Mount DW (2008) Using BLOSUM in sequence alignments. Cold Spring Harb Protoc 2008(6):pdb.top39

38. Taherzadeh G, Zhou Y, Liew AWC, Yang Y (2016) Sequence-based prediction of protein-carbohydrate binding sites using support vector machines. J Chem Inf Model 56(10):2115-2122

39. Meiler J, Müller M, Zeidler A, Schmäschke F (2001) Generation and evaluation of dimension-reduced amino acid parameter representations by artificial neural networks. Mol Model Annu 7(9):360-369

40. Narjeskhatoon Habibi1* SZMH, ANaMRS, 3,4: A review of machine learning methods to predict the solubility of overexpressed recombinant proteins in Escherichia coli. 2014. https://bmcbioinformatics.biomedcentral.com/artic les/10.1186/1471-2105-15-134

41. Altschul SF, Madden TL, Schäffer AA, Zhang J, Zhang Z, Miller W, Lipman DJ (1997) Gapped BLAST and PSI-BLAST: a new generation of protein database search programs. Nucleic Acids Res 25(17):3389-3402

42. Mirdita M, von den Driesch L, Galiez C, Martin MJ, Söding J, Steinegger M (2017) Uniclust databases of clustered and deeply annotated protein sequences and alignments. Nucleic Acids Res 45(D1):D170-D176

43. Heffernan R, Yang Y, Paliwal K, Zhou Y (2017) Capturing non-local interactions by long short-term memory bidirectional recurrent neural networks for improving prediction of protein secondary structure, backbone angles, contact numbers and solvent accessibility. Bioinformatics 33(18):2842-2849

44. Emerson IA, Amala A (2017) Protein contact maps: a binary depiction of protein 3D structures. Phys A 465:782-791

45. Kipf TN, Welling M. Semi-supervised classification with graph convolutional networks. arXiv preprint. arXiv:1609.02907; 2016

46. Zheng $S$, Yan X, Yang Y, Xu J (2019) Identifying structure-property relationships through SMILES syntax analysis with self-attention mechanism. J Chem Inf Model 59(2):914-923

47. Lin Z, Feng M, Santos CNd, Yu M, Xiang B, Zhou B, Bengio Y. A structured self-attentive sentence embedding. arXiv preprint . arXiv:1703.03130; 2017.

48. Kingma DP, Ba J: Adam. A method for stochastic optimization. arXiv preprint. arXiv:1412.6980; 2014

\section{Publisher's Note}

Springer Nature remains neutral with regard to jurisdictional claims in published maps and institutional affiliations. 